\title{
Research on Product Conceptual Design Based on Integrated of TRIZ and HOQ
}

\author{
Jianmin Xie, Xiaowo Tang, and Yunfei Shao \\ School of Management, University of Electronic Science and Technology of China, \\ 610054 Chengdu, China \\ \{Jianmin.Xie, Xiaowo.Tang, Yunfei.Shao, xiejm\} @uestc.edu.cn
}

\begin{abstract}
The conceptual design determines the success of the final product quality and competition of market. The determination of design parameters and the effective method to resolve parameters contradiction are the key to success. In this paper, the concept of HOQ products designed to determine the parameters, then using the TRIZ contradiction matrix and inventive principles of design parameters to solve the problem of contradictions. Facts have proved that the effective method is to obtain the product concept design parameters and to resolve contradictions line parameters.
\end{abstract}

Keywords: TRIZ, QFD, HOQ, Conceptual Design.

\section{Introduction}

The competition is becoming more fiercely, the change of customer product preferences is speeding up, making enterprises must continue to introduce new product. On the innovative design of new products has become a bottleneck in the development of many enterprises. The concept of product design is the design of new products in the important aspects of the conceptual design parameters, as well as to determine the parameters of an effective solution to the conflict is the concept of design is the key to success. House of Quality about QFD (House of Quality, referred to as HOQ) through the customer demand for the product concept to determine the design parameters, TRIZ contradiction matrix and the use of the principle of the invention can solve the technical contradiction between the parameters. Therefore, the integration of TRIZ and QFD is to address the concept of product design parameters were obtained, as well as parameters to resolve the conflict effectively.

\section{Access of Conceptual Design Parameters}

The parameters of product conceptual design are obtained by HOQ, the HOQ are the core of QFD. The theory can give the needs of customers to enterprises, according to the wishes of the customer's preferences designed to produce products that meet the requirements. The essence of HOQ is a system to ensure that the methods will make customers, market demand through the various stages of product development and 
accurately into the related technical parameters, so that the enterprise managers and staff to clear the track of products in the early customers to the level of demand for operating activities, as well as detailed instructions.

\subsection{House of Quality about QFD}

House of Quality (HOQ) is the core to driving the entire QFD (Quality Function Deployment), it is a large matrix, composed by seven different parts (Figure 1).The seven parts are:

1. Customer needs. That is VOC; they can be usually indicated by cohesion map and express tree. Different products have different customer demands. For example, for motor vehicles, customer needs may be easier to open the door; for banks, customer needs may be no need to queue up for cash. QFD is used to deploy (deploy) the voice of customer, rather than used for the collection of the voice of customers.

2. Product features. They can be also indicated by cohesion map and express tree. The means of product features are designed to meet our customer needs; product characteristics also differ among products. Such as the car doors, product features may be determined by how much the power to close the door; such as the mower, product features may be the rotational axis of the thrust required. Product features must be use the standardized statement. QFD uses the customer needs to generate the Product Characteristics.

3. The importance of customer needs. Not only do we need to know what the customer needs, but also know that these essential requirements for the degree of customers.

4. Plan matrix. The matrix contains one competitive analysis of a main competitor product. Matrix including the three, respectively, for the existing products on behalf of the required improvements (improvement rate), improved the possible increase in sales (sales points), as well as scores for each customer needs.

5. Customer demand and product characteristics of the relationship. This is the matrix of the body (middle part), indicating that the product characteristics of the various contributions to customer needs and the extent of the impact.

6. Features and characteristics of the relationship. In general, one change in the characterization often affects the other characteristics. This effect is usually negative, that is, a characteristic often leads another characteristic to improve the deterioration. Relationship between the properties so that we can distinguish between the effects of these characteristics, in order to achieve a compromise.

7. Target. This is result came form the above-mentioned part of the impact of product characteristics.

8. The House of Quality was consisted by the broad following matrix components: WHATS matrix, indicates need what; HOWS matrix, indicates how to do by need; correlation matrix, indicates the relationship of WHATS subparagraph of; HOWS The relationship between the matrix, indicates the relationship of every item in HOWS matrix; evaluation matrix, indicates HOWS organization or the cost of the technical evaluation of the situation; the feasibility of competitive or competitive or comparative analysis. 

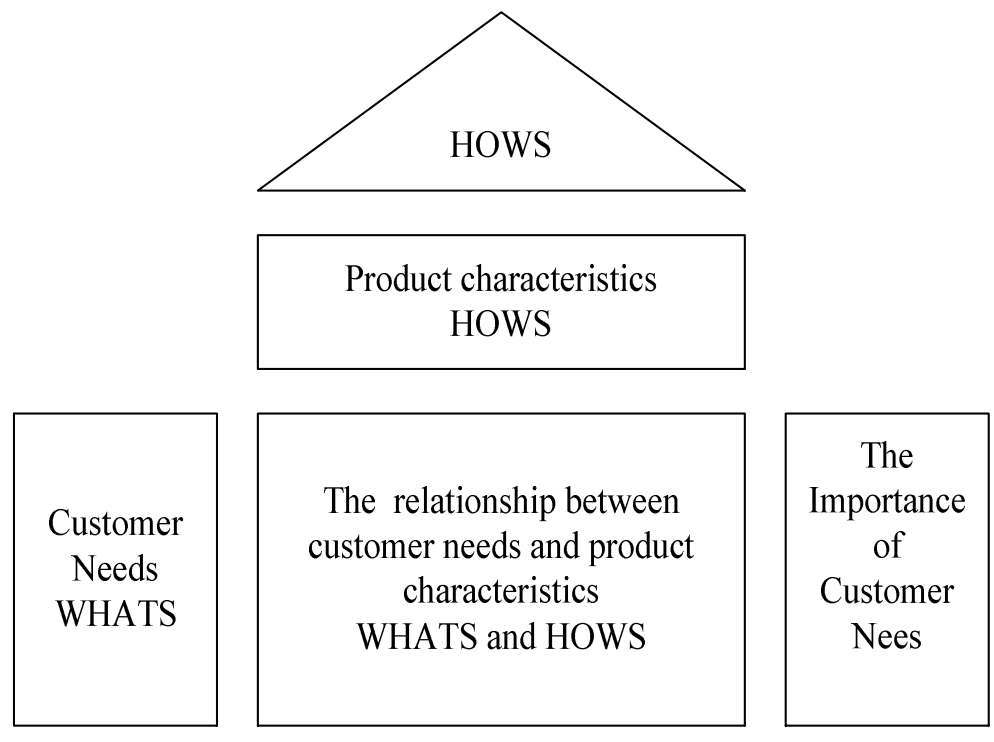

\section{HOWS Evaluation}

Fig. 1. Hows of Quality about QFD

\subsection{Customers Demand Matrix}

The acquisition of concept of product design parameters is achieved through the construction of QFD to the quality of housing.

Set up the number of the user need about one product is $\mathrm{N}$. The matrix of user need as C:

$$
C=\left[\begin{array}{l}
C_{1} \\
C_{2} \\
\vdots \\
C_{n}
\end{array}\right]
$$

$C_{1}$ expresses the first customer's needs; $C_{n}$ expresses the number $\mathrm{N}$ customer's needs.

\subsection{Technical Needs Matrix}

The choice of technical needs by technical engineers based on the user's specific needs to develop, usually to determine the technical requirements are based on the technical characteristics of similar products, using the analog method. Set up the 
number of technical needs according to a set of user demand for product is $M$, the technical needs matrix is:

$$
T=\left[\begin{array}{llll}
T_{1} & T_{2} & \ldots & T_{m}
\end{array}\right]
$$

$T_{1}$ expresses the first technology needs; $T_{m}$ expresses the number $\mathrm{N}$ technology needs.

\subsection{Translation between the Matrixes of Customer Needs and Technology Needs}

The relationship of matrix that user needs and technology needs of between the expressions is as follows:

$$
T=\left[\begin{array}{cccc}
C_{1} T_{1} & C_{1} T_{2} & \cdots & C_{1} T_{m} \\
C_{2} T_{1} & C_{2} T_{2} & \cdots & C_{2} T_{m} \\
\vdots & \vdots & \vdots & \vdots \\
C_{n} T_{1} & C_{n} T_{2} & \cdots & C_{n} T_{n}
\end{array}\right]
$$

$C_{i} T_{i}$ indicates the relationship between one of the first $i$ users need to demand for the first $j$ technical parameters, the relationship of a set can be used to indicate the degree of correlation between them, $C_{i} T_{j}=\left\{0 \leq C_{i} T_{j} \leq 1\right\}$. Among them, 0 expresses not relevant, approximation of its value 1 the more that the relationship between the degree of the bigger one.

\subsection{Technical Relativity Matrix}

Technical relativity matrix indicates the various technical parameters of the relationship between symmetry can be expressed as a matrix:

$$
T R=\left[\begin{array}{cccc}
T R_{11} & & & \\
T R_{21} & T R_{22} & & \\
\cdots & \cdots & \cdots & \\
T R_{m 1} & T R_{m 2} & \cdots & T R_{m m}
\end{array}\right]
$$

$T R_{i j}$ indicates one of the first parameter $i$ and $j$ parameters of the relationship between the collection of a relationship can be said, that negative correlation $-1,0$ said not related, 1said positively related.

From the above matrixes, the demand of technical parameters can be achieved by the user's needs, and to identify the parameters exist between the technical contradictions, however, solution of conflicts must use TRIZ theory among 39 common parameters, contradiction matrix and 40 principles of the invention. 


\section{Technical Design Parameters of the Settlement of Conflicts}

Design parameters of the conflict Technical through the contradictions among the TRIZ matrix algorithm optimization and fuzzy contradictions among the matrix of the principles of the invention to solve the problem. TRIZ is a well-known inventor Altshuller the former Soviet Union and its partners in the analysis of a large number of patents on the basis of summing up of a variety of technological development to follow the evolution of the model laws and works to solve a variety of contradictory rules and principles of innovation. The conflict matrix of which 40 principles of the invention is the core of the TRIZ theory.

\subsection{TRIZ Matrix of Contradictions}

Altshuller through a detailed study of a large number of patents, summed up the project to extract the commonly used expression in the field of system performance of 39 general parameters of the project and to narrow it down to 2 when the parameters of any conflict, which should be adopted to resolve the principles of the invention. These principles of the invention that is, the 40 principles of the invention. Altshuller parameters of the project will also conflicts with the principles of the invention corresponds to the establishment of relations, finishing a $39 \times 39$ matrix, that is, Altshuller matrix of contradictions.

The line and row uses the 39 general parameters of the project's name and serial number. The second row is the improve parameters and the first line is deterioration of the parameters. Matrix box in the middle of each square in the figure for the number of TRIZ recommended by the project corresponds to resolve contradictions in the invention of the principle of numbers. 45 degrees diagonal grid (black belt "+" box), are the same name Engineering parameters as the corresponding box, expressing contradictions are physics ones rather than engineering contradictions.

Table 1. TRIZ matrix of contradictions

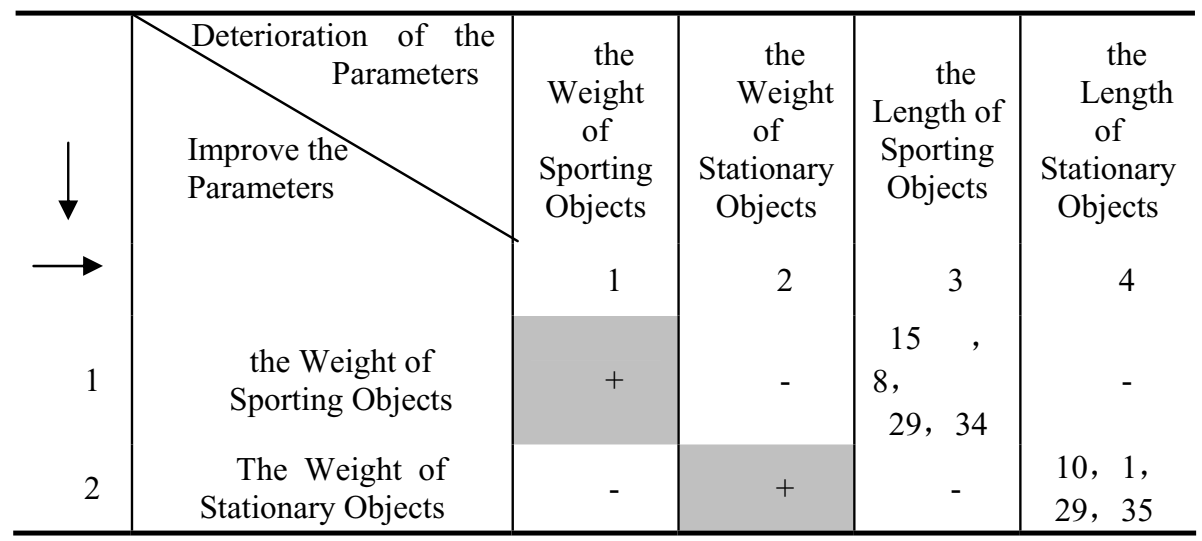




\subsection{Parameters of the Framework to Resolve Conflicts}

The use of TRIZ methods and tools of these parameters to resolve the contradictions in the framework map as shown in Figure 2:

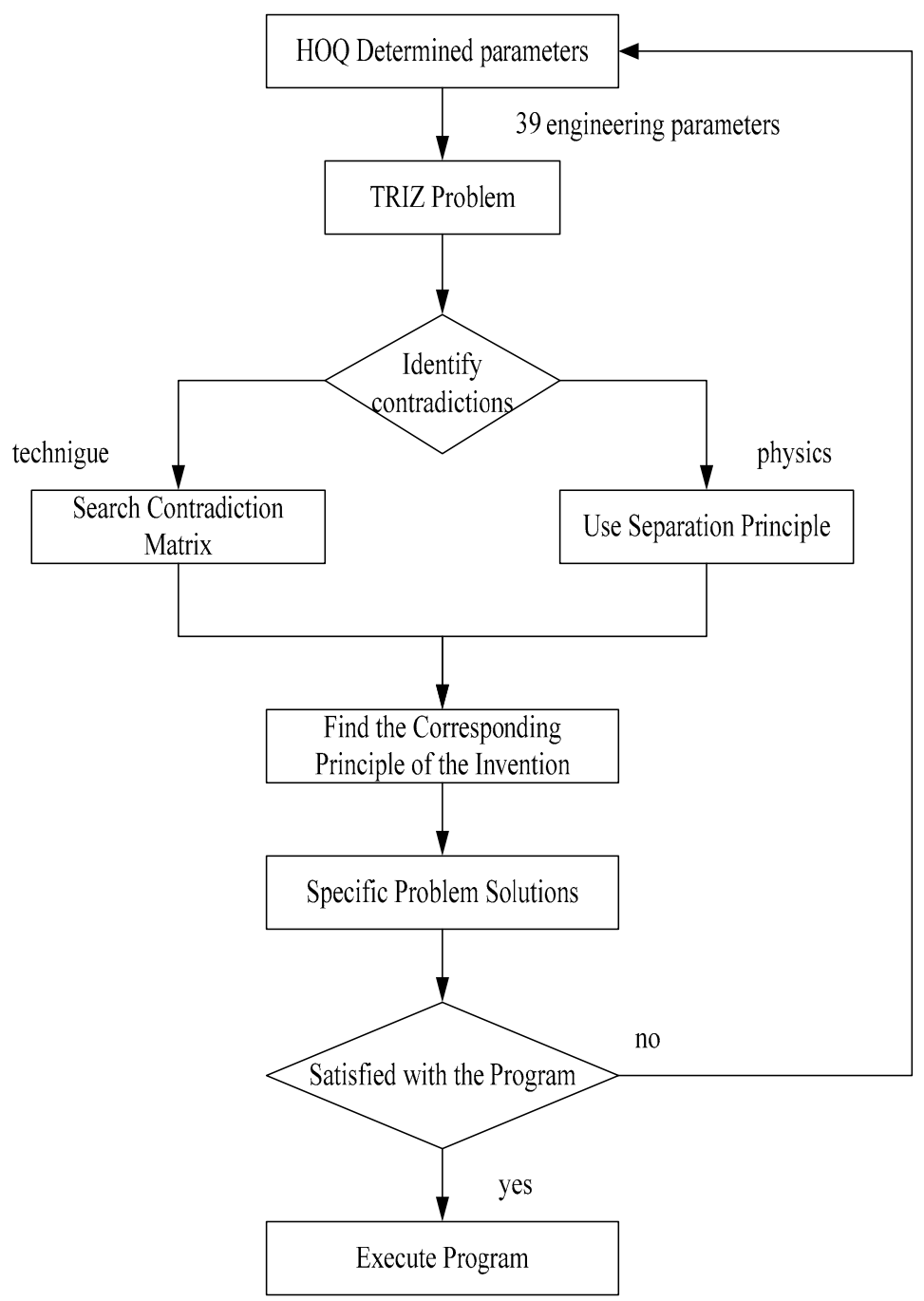

Fig. 2. The framework of using TRIZ to resolve parameters contradictions

The process of using TRIZ to resolve parameters contradictions can be described as: first of all, through the concept of HOQ products designed to determine the technical parameters, then uses some of 39 common engineering parameters to express problem to be resolved, so a specific problem will be changed into the TRIZ's problem; second, to determine the TRIZ problem are technical conflicts or physical 
contradictions. If it is technical contradictions, conflicts on the use of matrix from the 40 principles of the invention which is suited to find the principle, if it is physical contradictions, we can use the separation principle to determine the adapt ed principles of the invention; Finally, through the invention of the principle to find the incidence of specific problems, and the evaluation of the program, if satisfied with the program feasible, we can execute the program; if the program is not feasible, we must duplicate all the steps until you find satisfaction with the feasible solution as so far.

\section{Conclusion}

This article provides the solutions with the use of TRIZ and a HOQ integrated to solve product design parameters of the contradictions. TRIZ has a very good product design parameters to resolve contradictions in the tools and methods, however, parameters of acquisition and analysis of the unavailability of appropriate methods and tools to rely on people's experiences are summarized and a qualitative description of the problem to the implementation. Therefore, in order to better use of TRIZ to achieve product design innovation, and the urgent need for other methods or tools to solve this problem, through the QFD house of quality to fully understand customer needs, we can found a very good innovation to be resolved and accurately describe the problem. Therefore, TRIZ and QFD could be integrated as a scientific and innovative ways to realize the concept of Product Design Innovation.

\section{Acknowledgments}

The authors thank the project of Tech-innovation Methods Integration Research and Generalizing Application (NO. 2007FY140400), the project of Research on enterprises Total Innovation Management (NO. 2008ZR0015) and the project of Research on Innovative Method Based on Integrated of TRIZ and QFD (NO. 08SB007).

\section{References}

1. Karen, G.: Altshuller Father of Innovation - the Contradiction of TRIZ. TRIZ Journal (November 2002)

2. Moehrle, M.G.: What is TRIZ? From Conceptual Basicsto a Framework for Research. Creativity And Innovation Management (1) (2005)

3. Cohen, L.: Quality function deployment: how to make QFD work for you, pp. 11-13. Addison-Wesley Publishing Company, Massachusetts (1995)

4. Charles, V.T., Amy, J.C., Trappey, S.H.: A Computerized Quality Function Deployment Approach for Retail Services. Computers in. Engng 30(4), 611-622 (1996)

5. Taeho, P., Kim, K.: Determination of an optimal set of design requirements using house of quality. Journal of Operations Management 16, 569-581 (1998)

6. Chang, H.T., Chen, J.L.: The conflict-problem-solving CAD software integrating TRIZ into eco-innovation. Advances in Engineering Software 35, 553-566 (2004)

7. Sullivan, L.P.: Quality function deployment. Quality Progress 19(6), 39-50 (1986)

8. Fung, R.Y.K., Fung, J., Tang, et al.: Product design resource optimization using a non-linear fuzzy quality function deployment model. International Journal of Production Research 40(3), 585-599 (2002) 